\title{
Una contribución realista-crítica para un programa interdisciplinario sobre racismo
}

[A critical-realist contribution to an interdisciplinary program on racism]

\author{
Matías Jaramillo ${ }^{1}$ (D), Rocío Fernández ${ }^{2}$ (D) y Lidia Yáñez ${ }^{3}$ (D) \\ ${ }^{1}$ Universidad de Chile; ${ }^{2}$ Universidad de Cambridge, Reino Unido; \\ ${ }^{3}$ Universidad de Manchester, Reino Unido
}

\begin{abstract}
Resumen
Las investigaciones sobre migraciones contemporáneas han aumentado constantemente en Chile desde los años 90. También ha habido una paulatina emergencia de estudios sobre racismo con un enfoque en sus causas y efectos. Recogiendo planteamientos que insisten en la necesidad de enfoques inter, multi, y transdisciplinarios para estudiar estas temáticas, este trabajo propone la formulación de un programa interdisciplinario sobre el racismo para la articulación de saberes basados en el enfoque realista-crítico como metateoría de la sociedad. Se plantea que las definiciones ontológicas, epistemológicas y metodológicas de esta metateoría son altamente competentes para la interdisciplinariedad y se sitúa en el contexto actual de estudio del racismo en el patrón migratorio contemporáneo de Chile.
\end{abstract}

Palabras clave:racismo, realismo crítico, interdisciplinariedad, migraciones.

\begin{abstract}
Research on contemporary migration has increased steadily in Chile since the 1990s. There has also been a gradual emergence of studies on the causes and effects of racism. Bringing together approaches that insist on the need for inter-, multi-, or transdisciplinary approaches to the study of these issues, this article proposes the formulation of an interdisciplinary program on racism in Chile and the articulation of knowledge based on the critical-realist approach as a meta-theory of society. We propose that the ontological, epistemological, and methodological definitions of this meta-theory are highly competent in terms of interdisciplinarity and situated in the current context of the study of racism within contemporary migratory patterns in Chile.
\end{abstract}

Keywords: racism, critical realism, interdisciplinarity, migrations..

Contacto: La comunicación sobre este artículo debe ser enviada a Matías Jaramillo, email matijaramillorojas@gmail.com

Financiamiento: Esta investigación contó con financiamiento de la Agencia Nacional de Investigación y Desarrollo de Chile (ANID) a través de la Subdirección de Capital Humano / Becas Chile Doctorado 2020 - 21201772, Becas Chile Magíster 2019 - 72200421 y 2019 - 73200627. 


\section{INTRODUCCIÓN}

La ontología es una dimensión fundamental para establecer diálogos entre distintos saberes. González y Kröger (2020) lo demuestran al examinar la tensión que existe entre las comprensiones sobre los bosques nativos que tenían, por un lado, las comunidades locales y, por otro, los interventores con perspectiva global, lo que imposibilitaba la aplicación de una política forestal compartida. Con ello, mostraron la primacía del enfoque positivista y el tecnicismo en el abordaje de los bosques frente a saberes y concepciones de las comunidades que ni siquiera los objetivan en palabras. Así, los autores proponen una apertura onto-epistemológica para el diálogo entre distintas concepciones del mundo, en la que se manifiestan diferentes conflictos, como es con la primacía de los enfoques técnicos-positivistas.

Para promover un enfoque interdisciplinar que tenga una noción fuerte de la ontología y que pueda subsanar las debilidades en la proyección de programas de investigación interdisciplinares en ciencias sociales, es posible identificar herramientas teóricas y reflexivas en la perspectiva del realismo crítico (RC). En ese sentido, Bhaskar et al. (2018) plantean que una teoría interdisciplinaria para la investigación debe considerar una ontología tal como han sido considerados los aspectos epistemológicos.
Es decir, ir más allá del cómo medir, hacia la comprensión del ser a medir. $\mathrm{Al}$ asumir una ontología realista para la interdisciplina, se identifican múltiples mecanismos en el dominio de lo real que al ejercer sus poderes van dando forma a los fenómenos complejos que se estudian. De lo contrario, y si solo se considera lo epistemológico, surge el riesgo de caer en una jerarquización de aplicaciones metodológicas y técnicas como traducción del ejercicio interdisciplinario. Por lo anterior, la propuesta de este trabajo es el estudio del racismo desde un enfoque interdisciplinar. Esto implica la emergencia de un campo determinado dentro de los estudios migratorios, con preguntas que no respondan específicamente a idiosincrasias de una disciplina dominante, si no que más bien converjan en una perspectiva común. En este contexto, este ensayo propone una reflexión desde las concepciones del realismo crítico para pensar un campo de estudios interdisciplinar sobre el fenómeno del racismo en Chile. El racismo, un fenómeno complejo de las sociedades, no sólo modernas, tiene un estatuto problemático tanto teórica como políticamente. Por lo mismo, avanzar hacia una definición de campo de estudio no hegemonizado por una única disciplina puede proporcionar mejores formas científicas para explicar sus mecanismos operativos. 


\section{ONTOLOGÍA SOCIAL, EPISTEMOLOGÍA Y METODOLOGÍA REALISTA CRÍTICA}

Siguiendo a Archer (2009), cualquier investigador social moviliza una ontología, aunque no sea manifiesta ni sea consciente de ello. Esto es relevante pues en cualquier campo de estudio debe relacionarse la naturaleza de lo que existe con la forma en que se estudia. Es decir, lo que se asume que existe, influye necesariamente en las consideraciones sobre cómo debe ser explicado. Por ello, desde el $\mathrm{RC}$, la ontología social ocupa un rol regulador en dos sentidos: se identifica qué hay que explicar (entidades existentes) y se descartan explicaciones (entidades no existentes). De este modo, "tal clase de consistencia es un requerimiento general y usualmente demanda ajustes continuos en ambas direcciones entre ontología y metodología para conseguirla y sostenerla como tal" (Archer, 2009, p. 49). Entonces, para una investigación social en que descripción y explicación sean coherentes e involucre distintos saberes, se debe definir tanto la ontología social como los programas metodológicos asociados. Estos serían los fundamentos propuestos para la formulación de programas interdisciplinares desde el enfoque denominado metateórico del RC. Según Bhaskar (1998) las definiciones ontológicas y epistemológicas de la investigación social deberían superar las perspectivas reduccionistas del positivismo y la hermenéutica debido a tres razones fundamentales. Primero, la realidad es preexistente, su estatuto es independiente del conocimiento que tengamos de ella. Segundo, la realidad está conformada por múltiples estructuras estratificadas en distintos niveles de profundidad. Tercero, las estructuras sociales implican a humanos actuando y están también basadas en ideas, teniendo una especificidad histórica y espacial o territorial. Por lo tanto, el estudio de los fenómenos complejos y multivariables como el racismo puede tener una conducción en programas de investigación teniendo en cuenta los elementos anteriores, pues no todas las disciplinas están habilitadas para aprehender la totalidad del fenómeno ni son conscientes de todos sus mecanismos; asimismo, existen algunos enfoques más pertinentes que otros para abordar la agencia humana o los mecanismos estructurales.

La ontología del RC se basa en una estratificación de la realidad social en tres niveles: el nivel de lo empírico, de lo actual, y de lo real (Bhaskar, 1998). Lo empírico, que se conforma de eventos medibles que constituyen la interpretación y experiencia humana. Es decir, en este nivel ocurren las ideas, acciones, sentidos, y significados. Lo actual, involucra la ocurrencia real de estos eventos, independiente de su interpretación que tiende a diferir y que depende de la activación de ciertos poderes. Finalmente, el nivel de lo real se refiere principalmente a estructuras y mecanismos causales los cuales constituyen las propiedades inherentes de los objetos o estructuras que producen eventos al nivel de lo empírico.

A nivel epistemológico, el RC plantea la existencia de una realidad independiente de la conciencia de los individuos que la habitan, cuya capacidad de ser comprendida es constreñida y limitada. La realidad tiene dos dominios diferentes mutuamente incrustados. El primero se refiere al dominio intransitivo, que contiene las entidades, cosas, 
estructuras, y tendencias reales. El segundo se refiere al dominio de la transitividad, que contiene lo que se puede conocer de esta realidad. Este segundo dominio es un producto social, históricamente elaborado y en permanente cambio. Por tanto, el $\mathrm{RC}$ asume un dominio de realismo ontológico del que surge un relativismo epistemológico.

Así, el RC trasciende del nivel empírico de la realidad, superando el uso sesgado de los datos y dotándole a la abstracción teórico-conceptual un papel importante en la identificación y explicación de los mecanismos causales y los poderes estructurales y agenciales que operan en ella. Por lo tanto, es en la identificación de esos mecanismos causales que configuran un fenómeno complejo en donde la interdisciplinariedad puede tener un sentido científico. Si bien para el realismo crítico la realidad trasciende a la consciencia de ella, la ciencia es un producto histórico y social, por lo tanto, epistemológicamente es abierta, relativa y provisional. Es decir, dentro de la potencial comunidad interdisciplinaria, los ajustes del programa de investigación deben basarse en una reflexión en torno a los modos de producción científica en relación con su contexto de producción. En términos metodológicos, Archer (1997, 2009) plantea dos características fundamentales para la construcción de un programa de investigación en ciencias sociales que vincule lo anterior con la empírea: el dualismo analítico y el enfoque morfogenético o morfoestático. El dualismo analítico significa que existen dos entidades en la realidad social, la agencia y la estructura, que son irreductibles entre sí y deben ser separadas analíticamente para el estudio de la sociedad. La separación es analítica debido a que la sociedad es inseparable de los agentes y de su propia existencia. La sociedad es tanto transformable por las acciones de los agentes -lejos de una inmutabilidad, como causa de afectación a las acciones humanas que la buscan transformar y reproducir. En ese sentido, lo que se estudia es el juego mutuo entre los poderes causales entre agencia y estructura sin conflacionarlas, es decir, reducir una de las dos entidades a la otra.

El marco morfogenético o morfoestático ofrece una vinculación entre ontología social y teoría práctica, enfatizando el juego mutuo entre agencia y estructura. Esto porque considera que las formas sociales de cultura y estructura son preexistentes a las acciones de los sujetos y autónomas de ella. Así, se deben analizar separadamente condiciones y acciones, considerando por lo tanto la distinción temporal de transformación y reproducción mediante el juego mutuo de la agencia y la estructura (Archer, 2009). Aquí, las estructuras sociales tienen una formación temporal más larga que el tiempo de las acciones de los individuos y aunque son en la realidad inseparables, analíticamente deben considerarse estas diferencias temporales. Transversalmente a esto, la noción de emergencia tiene importante implicancia para el realismo crítico. Esto quiere decir que Archer muestra claramente la dualidad ontológica de estructura y acción en las propiedades emergentes autónomas (Mascareño, 2008).

Considerando que todo fenómeno social es producto de relaciones de otras entidades, emergencia significa que las propiedades de los distintos fenómenos no se pueden reducir a los aspectos de la realidad que les dieron origen (Sayer, 2000). Por ejemplo, respecto de actitudes racistas, se debe considerar la interacción de las acciones y 
reflexividad de los agentes con aquellos aspectos culturales producidos y reproducidos por los medios de comunicación, pero no sobredeterminar estas actitudes por las ideologías a las que se ven expuestos. Esto es fundamental para un estudio interdisciplinar del racismo porque los fenómenos deben ser entendidos considerando las propiedades emergentes de las entidades socioculturales que los generan. La forma de estudiar la reflexividad de los agentes cuando ejercen racismo involucra aspectos psicológicos y morales que pueden ser abordados por la psicología y la sociología, mientras que la forma de estudiar las ideologías transmitidas por los medios de comunicación involucra la generación de un discurso y su reproducción e interpretación mediante interacciones, lo que puede ser abordado por áreas como la antropología o estudios culturales; pero también por la economía si es que se busca entender las condiciones materiales en que se producen estos discursos.

En síntesis, la interdisciplinaridad debe ser guiada tanto por una sólida consideración ontológica del fenómeno social al que nos enfrentamos, así como también por el conocimiento producido acerca de este, en tanto práctica social. En este sentido, el $\mathrm{RC}$ ofrece un marco para resguardar y poner en diálogo la coherencia entre definiciones epistemológicas y metodológicas con las definiciones ontológicas desde las cuales pensar la interdisciplinariedad.

\section{Un estudio realista crítico del racismo}

El concepto de racismo es problemático en cuanto a su estatus conceptual y político. Balibar (2005) se pregunta por qué un conjunto de discursos que aíslan, estigmatizan, y discriminan a un grupo humano son considerados racistas, y concluye que la categoría de racismo ha alcanzado un punto de descomposición y deconstrucción. Casáus (2018), plantea que por más que se considere superado el problema del racismo en el mundo, éste se ha transformado en función de la coyuntura política, contexto histórico, y crisis de dominación. Así, aún queda establecer los mecanismos para erradicar el racismo, pues "la exploración de la complejidad del racismo dista mucho de ser un tema concluido, pero no puede obviarse que hay una necesidad cada día más apremiante de combatirlo" (Velasco, 2007, p. 132). Siguiendo a Troyano (2010), hay múltiples definiciones del racismo, ninguna de las cuales es precisa, ya sea porque no lo definen de buena manera y/o que pretenden definir algo que no es un mismo fenómeno. Por lo tanto, un programa interdisciplinario tiene la potencialidad de continuar con los esfuerzos de componer el concepto (Wimmer, 1997), sin caer en riesgos como considerarlo novedoso cuando no lo es (Aguilera, 1992) y superando su lugar periférico que lo ha acompañado desde su emergencia en el campo sociológico desde el siglo XX (Pineda, 2016). Una definición operativa de racismo inspirada en Foucault (1996) y Balibar y Wallerstein (1988), sería una construcción histórica de otredades desde el Estado reproducido en instituciones, organizaciones y familias para la higienización y protección del nosotros, basadas en la idea de una etnicidad ficticia y comunidad imaginada en forma de desigualdad estructural que intersecta con la clase y el sexogénero para su reproducción contemporánea. Desde la perspectiva del RC (Carter, 1998, 2000, 2003; Carter \& Virdee, 2008; Campos, 2017), los programas de investigación deben estudiar las 
causas, dinámicas y consecuencias del racismo desde una integración analítica de las tres principales dimensiones que se ha estudiado, aunque de forma conflacionista: sistémico-estructural, idea-ideologías, prácticas-acciones-actitudes. En consecuencia, una articulación del estudio del racismo desde el RC tiene tres ventajas. (a) La diferenciación entre integración entre sistemas e interacción social (o entre integración sistémica e integración social); (b) la identificación de diferencias entre personas y estructuras y; (c) el reconocimiento del carácter emergente del racismo como consecuencias intencionadas y no intencionadas entre ideas y prácticas racistas (Carter, 1998). Por ejemplo, considerar que hay dos niveles de integración de población inmigrante permite hacer la distinción entre la integración dada por las regulaciones normativas y legislativas dada por interacciones sociales.

Una explicación realista-crítica podría comenzar reconociendo que las ideas sobre la raza son elementos dentro de un ámbito cultural independiente de cualquier punto de vista particular. Se deben considerar los esquemas conceptuales y los sistemas de creencias como propiedades emergentes culturales (Archer, 1997) irreducibles al nivel de los individuos y que de hecho están abiertas a interpretaciones $\mathrm{y}$ debates. En este sentido, "las teorías, ideas y conjeturas pueden postularse como entidades relativamente independientes con interconexiones lógicas contingentes o necesarias" (Carter, 2003, p. 5) posibilitando una explicación de las ideas raciales. Por lo tanto, la propuesta es no subsumir el racismo a ideas racistas (Carter, 2000) cuidando especialmente los postulados epistemológicos expuestos anteriormente, considerando la autonomía de las estructuras.

\section{Multi-trans-interdisciplinariedad}

Distintos esfuerzos han relevado la necesidad de un avance en los programas de investigación que superen el cierre disciplinar para el estudio de las migraciones contemporáneas y los efectos que ha tenido en la sociedad receptora. Tapia y Liberona (2018) abordaron la transdisciplinariedad en los estudios migratorios en Chile en concordancia con Cano y Soffia (2009), quienes sistematizaron el aumento de producción académica sobre migraciones en Chile, o por Stefoni (2017) a nivel sudamericano. Sin embargo, su propuesta transdisciplinar fue convocar a una escritura de un libro en que distintos investigadores de distintas disciplinas escribieran distintos capítulos. Queda por lo tanto el desafío de una producción que vincule esos saberes para proyectar una investigación interdisciplinaria ante la urgencia de estudiar la migración y el racismo en sus complejas manifestaciones.

Olmos y Martín (2020) discuten la cuestión metodológica, en términos de que el debate cuantitativo-cualitativo parece manifestar en este fenómeno de forma más clara su falsa dualidad: tanto la entrevista en profundidad como los cuestionarios estandarizados no darían cuenta del fenómeno en su conjunto y optar solo por uno de ellos es un riesgo. En cuanto a formulaciones transdisciplinares, Chávez (2017) propone la construcción de un marco teórico transdisciplinar para el estudio de las migraciones transnacionales dada la compleja 
realidad a investigar que suponen los procesos migratorios. Propone categorías y variables a estudiar para trascenderlos límites disciplinares en búsqueda de marcos analíticos más diversos y complejos: categorías como relaciones socioculturales y variables como solidaridad, reciprocidad e intercambio social, por ejemplo, consolidadas en dimensiones de estudio. Gall (2014) por su parte, aborda específicamente la transdisciplina en el racismo, operativizando este concepto desde los estudios interseccionales, pero a la vez, equiparando el estatus de transdisciplinariedad con el de interseccionalidad, pues "la interseccionalidad / interdisciplina es la base de la postura teórico-metodológica que creemos urgente profundizar en la investigación sobre el racismo" (Gall, 2014, p. 30).

Estos esfuerzos son relevantes porque generan propuestas de un programa de investigación sobre migración y racismo, pues siguiendo a Schmalz et al. (2019), los tres "modos de colaboración" sirven para propósitos específicos y se manifiestan de forma diferente y se caracterizan por: (a) Multidisciplinariedad mediante equipos colaborativos de diferentes campos que examinan una pregunta similar que es amplia. En la mayor parte del trabajo ejercen las disciplinas en paralelo, aunque pueden reunirse en ciertos puntos. (b) Interdisciplinariedad donde investigadores se informan mutuamente sobre sus perspectivas y transferencia de conocimiento a través de disciplinas. (c) Transdisciplinariedad en la cual se crea el propio espacio intelectual fuera de los límites de las áreas de investigación, dejando de lado las agendas disciplinares para abordar problemas multifacéticos y encontrar soluciones.

Price (2015) destaca las ventajas de un enfoque RC de interdisciplinariedad como respuesta a la tendencia predominante en este campo: la codisciplinary, pues, a su juicio, comete errores teóricos y epistemológicos como hipostasiar hechos, fetichizar las conjunciones constantes de eventos, y aplicar a sistemas abiertos epistemologías planteadas para sistemas cerrados. Frente a estos errores, una teoría RC de la interdisciplinariedad reconoce capas empíricas, actuales, y reales de los fenómenos sociales, por lo que puede proporcionar explicaciones más complejas y profundas que impliquen saberes de distintas disciplinas, cuestión requerida ante la identificación de problemas complejos que requieren el diálogo de múltiples saberes. Para que ello tenga un sentido operativo, proponemos como objeto de estudio y análisis comprender cuáles son los mecanismos estructurales, agenciales, e ideológicos del racismo y sus transformaciones a través del tiempo en un estudio que se observen de forma interdisciplinaria sus manifestaciones empíricas en distintos niveles emergentes de la realidad (i.e., individuos, organizaciones e instituciones). Esto es pertinente en la actualidad ante la complejización y dinamización del patrón migratorio chileno (Jaramillo, 2018) y la pluralidad de manifestaciones racistas (Instituto Nacional de Derechos Humanos [INDH], 2017). La complejidad del mundo exige que los sistemas de conocimiento cambien a enfoques holísticos y orgánicos que consideren diversos puntos de vista (Budruk \& Feldhaus, 2019). En ese sentido, lo que estructura el campo del racismo son las dimensiones transversales en campos y temáticas a estudiar, mientras que las disciplinas están al servicio de esas dimensiones internas y necesarias del racismo; no al revés. La coordinación sistémica de este esfuerzo interdisciplinar en el estudio de un fenómeno como el racismo puede tener curso ya que el $\mathrm{RC}$ 
opera como metateoría, es decir, constituye un tercer orden de análisis que se ocupa de examinar críticamente los corpus de conocimiento que los científicos sociales han logrado articular en el segundo orden de análisis y construcción de categorías que es el locus desde donde se generan las teorías explicativas y/o comprensivas del mundo fáctico (Toledo-Nickels, 2018, p. 204).

Por lo tanto, una definición realista de interdisciplinariedad que sea operativa para el racismo implicaría a investigadores de las disciplinas pertinentes para abordar la pregunta de investigación la cual integre "el análisis de estructuras, mecanismos y resultados en estos niveles mediante el uso del diseño y la metodología del estudio que son más apropiados para los niveles respectivos" (Danermark, 2019, p. 3). Entonces, un esfuerzo interdisciplinar de este tipo estaría formado por las siguientes fases: (a) Planificación (i.e., definición del racismo como un objeto de estudio interdisciplinar y la identificación preliminar de estructuras y mecanismos a incluir en la investigación). (b) Comprensión disciplinaria (i.e., sistematizar el conocimiento de estructuras, mecanismos, y contextos desde las disciplinas específicas, pero en diálogo entre ellas). (c) Comprensión interdisciplinaria (i.e., asimilar actitudes de apertura, respeto mutuo y habilidades complementarias para el desarrollo de un lenguaje común). (d) Teorización transfactual (la integración de conocimiento es fundamental para sobrepasar el nivel multidisciplinario y se realiza y tiene sentido al basarse en las tres fases anteriores). (e) Comprensión interdisciplinaria (como fusión efectiva de disciplinas, posee una propiedad emergente como investigación no pudiendo ser reproducidos por alguna disciplina en particular).

En este sentido, una sistematización de elementos emergentes en dimensiones transversales del fenómeno plantea el desafío de operacionalizar un esquema que pueda abrirse al debate y aportar a los distintos programas de investigación sobre racismo y migraciones contemporáneas. Con el RC como articulador se proponen definiciones analíticas cuyo propósito es atender a las dimensiones ontológicas del fenómeno sin fusionarlas a través de mecanismos sistémicos-estructurales (producidos y reproducidos a través de relaciones sociales institucionalizadas), ideológicosculturales (reproducidas a través de proposiciones ideacionales hegemónicas), de práctica-comportamientos-actitudinales (personas reales que poseen reflexividad y actúan articulando metas, recursos, y cursos de acción en un proyecto de vida; y pueden articularse en otros niveles agenciales). Por otro lado, se proponen definiciones operativas que, en términos metodológicos, debiesen observar el nivel empírico (i.e., manifestaciones observadas del racismo como discursos, violencias, desigualdades, etc.), actual (i.e., acontecimientos contingentes como el patrón migratorio, racialización basada en color de piel y nacionalidad, etc.) y real (i.e., mecanismos causales como la ideología de la blanquituddesarrollo, la estructura étnico-racial, etc.). De este modo, la Tabla 1 propone una serie de campos y temáticas a estudiar. 
Tabla 1. Propuesta de Objetivos, Campos y Temas Para el Estudio del Racismo

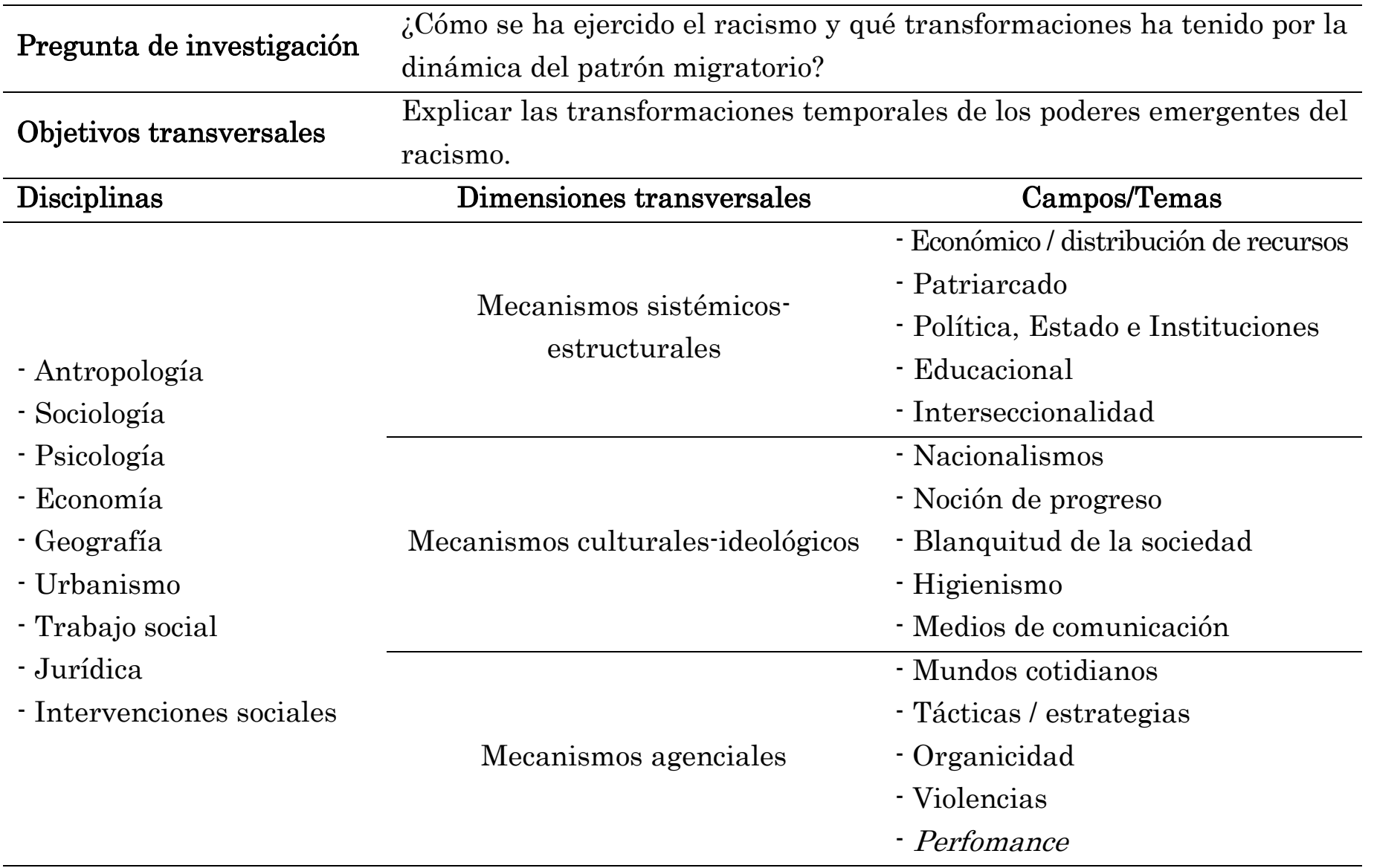

Sobre los campos temáticos propuestos en la Tabla 1, en la dimensión transversal sobre mecanismos sistémico-estructurales, un abordaje interseccional implicaría considerar cómo distintas estructuras como la clase social, sexo-género, y racialización interaccionan para producir una situación de desigualdad social específica. Una aproximación interseccional desde el RC, considera la autonomía y el mutuo moldeamiento de estos sistemas sociales de desigualdad, los cuáles se encuentran constituidos socio-históricamente (Walby et al., 2012). Esto ha sido estudiado, en la sexualización de mujeres migrantes afrocaribeñas en Chile (Belliard, 2015), donde procesos históricos y coloniales de racialización de cuerpos negros, en interacción con desigualdades sexo-genéricas, producen una sexualización de estas corporalidades en las interacciones cotidianas. Respecto a los mecanismos culturalesideológicos, se ha estudiado cómo se produce un discurso racista por los medios de comunicación para el caso de inmigrantes (Vial, 2015) y la población mapuche (Gallardo, 2015). Se ha considerado, la influencia de los medios de comunicación en la producción y legitimación de ciertas ideas, y cómo la concentración de los 
medios en ciertos sectores privilegiados de la sociedad excluye y estigmatiza a otros sectores marginalizados racializados. Sin embargo, el campo de estudio se beneficiaría por la interdisciplinaridad, ya que suele ser abordado desde el análisis del discurso de los medios de comunicación, sin considerar la interacción de aquellas ideas con los agentes, que no reciben pasivamente estas ideas, sino que las interpretan y modifican en sus interacciones.

Respecto del campo temático sobre los mecanismos agenciales, se han estudiado las experiencias de racismo cotidiano y el conocimiento de este por parte de jóvenes inmigrantes peruanos (Correa, 2014). Las situaciones de violencias cotidianas son producidas a través del estigma racista. Los jóvenes enfrentan estas situaciones con un conocimiento situacional del racismo, dándose como resultado la justificación y consecuente naturalización de estas violencias cotidianas, o el enfrentamiento de ellas desde discursos de nacionalismo que exacerban las diferencias. El establecimiento de un estigma social, que se produce históricamente y se reproduce a nivel de interacciones cotidianas, permite entender las violencias cotidianas que enfrentan estos jóvenes, y vincularlo con el rol que juega este estigma racista dentro de las construcciones de Estados-Nación.

\section{CONCLUSIONES}

En este trabajo se planteó que establecer una propuesta multi-inter-transdisciplinar en las ciencias sociales requiere de un diálogo particular en torno a definiciones ontológicas, que resguarde la coherencia con las definiciones epistemológicas y metodológicas. Por ello, se buscó reconstruir un campo interdisciplinario desde el RC insertándolo en el emergente campo del racismo. Como plantea Danermanrk (2019), hacer una investigación interdisciplinaria no es solo una cuestión de desarrollar nuevos conocimientos y contribuir a un mayor desarrollo de una agenda de investigación sobre un problema social. Si identificamos el racismo como un problema altamente complejo, los desafíos a los que enfrentan la investigación a nivel teórico y metodológico están en concordancia. Entonces, no se sostendría una explicación integral de la formación del racismo como fenómeno social únicamente desde una disciplina. Es probable que un trabajo interdisciplinar, puedan enfrentar los desafios epistemológicos de su estudio identificados por Correa (2016) al dar cuenta que se ha considerado a la inmigración como un problema y no las estructuras y mecanismos que lo vuelven problemático.

Una investigación interdisciplinaria sobre el racismo puede incorporar un horizonte normativo, en términos de discusión y erradicación de las manifestaciones del racismo en Chile. En muchos casos, la razón de ser de la investigación interdisciplinar es el deseo de intervenir y cambiar la realidad, es decir, de abolir o mitigar las condiciones negativas de la sociedad. Para ello, los fundamentos científicos del $\mathrm{RC}$, explicar la realidad es a la vez un prerrequisito para su transformación como un desafío constante y 
complejo para la formulación teórica (Bhaskar, 2003). Si una ciencia antirracista quiere desplegar sus facultades, es más probable que en la convergencia de saberes y en la emergencia de un campo de estudio pueda generar mayores efectos para la intervención en y la transformación de la sociedad.

\section{REFERENCIAS}

Aguilera, R. (1992). Para una sociología del racismo: Análisis comparativo de las pautas de matrimonio entre personas de raza blanca y negra. REIS: Revista Española de Investigaciones Sociológicas, 92(60), 47-61. http://www.reis.cis.es/REIS/PDF/REIS_060_05.pdf

Archer, M. (1997). Cultura y teoría social. Nueva Visión.

Archer, M. (2003). Being human: The problem of the agency. Cambridge University Press.

Archer, M. (2009). Teoría social realista: En enfoque morfogenético. Ediciones Alberto Hurtado.

Balibar, E. (2005). La construction du racisme. Actuel Marx (38), 11-28. https://doi.org/10.3917/amx.038.0011

Balibar, E., \& Wallerstein, I. (1988). Raza, nación, clase. IEPALA Editorial.

Belliard, C. (2015). Negritudes extranjeras en Chile. Significaciones y estereotipos sexogenérico racializados en torno a los inmigrantes afro-latinoamericanos en Santiago de Chile [Proyecto de memoria para optar al título de antropóloga social, Universidad de Chile]. Repositorio Académico de la Universidad de Chile. https://repositorio.uchile.cl/handle/2250/136044

Bhaskar, R. (1998). The possibility of naturalism. Routledge.
Bhaskar, R. (2003). Realismo crítico, relaciones sociales y defensa del socialismo. Revista VientoSur. https://vientosur.info/realismo-criticorelaciones-sociales-y-defensa-del-socialismo/ Bhaskar, R., Danemark, B., \& Price, L. (2018). Interdisciplinarity and wellbeing. A critical realist general theory of interdisciplinarity. Routledge.

Budruk, M., \& Feldhaus, A. (2019). Understanding place meaning through integrative research: Perspectives from the natural resource social sciences and the humanities. Journal of Leisure Research, 5(50), 461-478. https://doi.org/10.1080/00222216.2019.1615395

Campos, L. (2017). Racismo em três dimensöes: Uma abordagem realista-crítica. Revista Brasileira de Ciências Sociais, 32(95), 2-19. https://doi.org/10.17666/329507/2017

Cano, V., \& Soffia, M. (2009). Los estudios sobre migración internacional en Chile: Apuntes y comentarios para una agenda de investigación actualizada. Papeles de Población, (61), 129-167. https://rppoblacion.uaemex.mx/article/view/ 8535/7245

Carter, B. (1998). 'Dangerous phrases': Realism, 'race' and social science. Alethia, 1(2), 5-8. https://doi.org/10.1558/aleth.v1i2.5

Carter, B. (2000). Realism and racism. Concepts of race in sociological research. Routledge. 
Jaramillo, M., Fernández, R., \& Yáñez, L. (2021). Una contribución realista-crítica para un programa interdisciplinario sobre racismo. Revista de Sociología, $36(2), 88-100$. 529X.2021.65566

Carter, B. (2003). What race means to realists. En J. Cruickshank (Ed.), Critical realism. The difference that it makes (pp. 149-160). Routledge.

Carter, B., \& Virdee, S. (2008). Racism and the sociological imagination. The British Journal of Sociology, 59(4), 661-679. https://doi.org/10.1111/j.1468-4446.2008.00214.x

Casáus, M. (2018). El racismo y su proyección actual: ¿Un fenómeno nuevo o un problema sin resolver? Cuadernos de Trabajo Social, 31(1), 121-137.https://doi.org/10.5209/CUTS.55732

Chávez, G. (2017). El trabajo social y la transdisciplinariedad: Retos para estudiar la migración transnacional, la comunidad y la comunicación. Cuadernos de Trabajo Social, 31(1), 21-33. https://doi.org/10.5209/CUTS.55996

Correa, J. (2014) El conocimiento del racismo como conocimiento político: Experiencias de racismo cotidiano de jóvenes hijos e hijas de inmigrantes peruanos en Santiago de Chile. CLACSO.

Correa, J. (2016). La inmigración como "problema" o el resurgir de la raza. Racismo general, racismo cotidiano y su papel en la conformación de la Nación. En M. Tijoux (Ed.), Racismo en Chile. La piel como marca de inmigración (pp. 35-48). Editorial Universitaria.

Danermark, B. (2019). Applied interdisciplinary research: A critical realist perspective. Journal of Critical Realism, 18(4), 368-382. https://doi.org/10.1080/14767430.2019.1644983

Foucault, M. (1996). Genealogía del racismo. Editorial Altamira.
Gallardo, J. S. (2015). Análisis crítico del discurso y representación de los mapuches en la prensa escrita chilena. Lenguas $y$ Literaturas Indoamericanas, (17), 145-169. http://revistas.ufro.cl/ojs/index.php/indoame ricana/article/view/604

Gall, O. (2014). Interseccionalidad e interdisciplina para entender y combatir el racismo. Interdisciplina, 2(4), 9-34.

http://computo.ceiich.unam.mx/webceiich/do cs/revis/interV1-N04.pdf

González, N., \& Kröger, M. (2020). The potential of Amazon indigenous agroforestry practices and ontologies for rethinking global forest governance. Forest Policy and Economics, 118, Artículo 102257.

https://doi.org/10.1016/j.forpol.2020.102257

Instituto Nacional de Derechos Humanos. (2017). Situación de los derechos humanos en Chile (Informe anual). https://www.indh.cl/bb/wpcontent/uploads/2017/12/01_Informe-Anual2017.pdf

Jaramillo, M. (2018). Manifestaciones de Racismo en Chile. Perfiles y problematización [Tesis para optar al grado de Magíster en Ciencias Sociales con mención en Sociología de la modernización. Universidad de Chile]. Repositorio Académico de la Universidad de Chile.

https://repositorio.uchile.cl/handle/2250/176096

Mascareño, A. (2008). Acción, estructura y emergencia en la teoría sociológica. Revista de Sociología, (22), 217-256. https://doi.org/10.5354/0719529X.2008.14492

Olmos, A., \& Martín, P. (2020). Retos epistémicosmetodológicos en la investigación sobre racismo. 
Jaramillo, M., Fernández, R., \& Yáñez, L. (2021). Una contribución realista-crítica para un programa interdisciplinario sobre racismo. Revista de Sociología, 36(2), 88-100. 529X.2021.65566

En S. Sassone, B. Padilla, M. González, B. Matossian, \& C. Melella (Comps.), Diversidad, migraciones y participación ciudadana. Identidades y relaciones interculturales (pp. 25-42). Instituto Multidisciplinario de Historia y Ciencias Humanas.

Pineda, E. (2016). Periferias sociológicas: Discriminación racial y Afrodescendencia. Revista Espacio Abierto, 25(4), 109-116. https://www.aacademica.org/estherpinedag/8.pdf Price, L. (2015). Critical realist versus mainstream interdisciplinarity. Journal of Critical Realism, 13(1), 52-76. https://doi.org/10.1179/1476743013Z.000000 00019

Sayer, A. (2000). Realism and social science. Sage.

Schmalz, D., Janke, M., \& Payne, L. (2019). Multi-, inter-, and transdisciplinary research: Leisure studies past, present, and future. Journal of Leisure Research, 5(50), 389-393. https://doi.org/10.1080/00222216.2019.1647751

Stefoni, C. (2017). Panorama de la inmigración internacional en América del Sur. CEPAL; Naciones Unidas; IOM.

Tapia, M., \& Liberona, N. (Eds.). (2018). El afán de cruzar las fronteras. Enfoques transdisciplinarios sobre migraciones y movilidad en Sudamérica y Chile. RIL Editores; Universidad Arturo Prat. Toledo-Nickels, U. (2018). El esquema metateórico de Ritzer desde la metodología de los programas de investigación. Cinta de Moebio. Revista de Epistemología de Ciencias Sociales, (33), 204-218. http://doi.org/10.4067/S0717554X2008000300004

Troyano, J. (2010). El racismo. Consideraciones sobre su definición conceptual y operativa. RIEM: Revista Internacional de Estudios Migratorios, (1), 1-24. https://doi.org/10.25 115/riem.v0i1.360

Velasco, S. (2007). El racismo y las tres formas básicas de combatirlo. Cultura $y$ representaciones sociales, 2(3), 131-150. http://www.culturayrs.unam.mx/index.php/ CRS/article/view/514/521

Vial, F. (2005). Prensa escrita, racismo y xenofobia [Tesis doctoral, Universidad Andrés Bello]. Repositorio UNAB. http://repositorio.unab.cl/xmlui/handle/ria/3635 Walby, S., Armstrong, J., \& Strid, S. (2012). Intersectionality: Multiple inequalities in social theory. Sociology, 46(2), 224-240. https://doi.org/10.1177\%2F0038038511416164

Wimmer, A. (1997). Explaining xenophobia and Racism: A critical review of current research approaches. Ethnic and Racial Studies, 20(1), 17-41. https://doi.org/10.1080/01419870.1997.9993946

Manuscrito recibido: 09-04-2021 Manuscrito aceptado: 15-11-2021 